\title{
A True New Gene: An Invertebrate Antibody Igkappa Gene
}

\author{
${ }^{1}$ Michel Leclerc, ${ }^{2}$ Nadine Vincent, ${ }^{2}$ Magne Osteras and ${ }^{2}$ Patricia Otten \\ ${ }^{1} 556$ Rue Isabelle Romée, Sandillon, France \\ ${ }^{2}$ Fasteris CH-1228, Plan-les-Ouates, Switzerland
}

Received 2013-09-07, Revised 2013-09-23; Accepted 2013-09-24

\begin{abstract}
The sea star Asterias rubens reacts specifically to the antigen: Horse-Radish Peroxydase (HRP) and produces an «antibody» anti-HRP.We had presented a candidate IgKappa gene corresponding to this last one in 2013. We show now the gene called: «sea star IgKappa gene» in its specificity. We have to know, at the present time that, at our knowledge, the sea star doesn't react specifically to all the antigens.
\end{abstract}

Keywords: Invertebrate, Sea Star, Igkappa Gene

\section{INTRODUCTION}

A first experiment which was fundamental, described in 1973, the immune response to various antigens of an asterid: Asterina gibbosa. It indicated (Leclerc, 1973), more exactly, the specific immunocytochemical response to Horse-Radish Peroxydase (HRP) of the sea star.

The general idea that emerged from the experiments made in our laboratories was that Echinodermata (Leclerc and Brillouet, 1981; Delmotte et al., 2012), as exemplified by sea stars: Asterina gibbosa and Asterias rubens, possessed an immune system able to mount cellular and humoral-specific responses after stimulation with a foreign antigen. In 2011 sea star antibody was correlated to IgKappa genes (Leclerc et al., 2011) and in 2013 a «true» candidate IgKappa gene was found. Then, it was shown a «true» gene from this candidate.

\section{MATERIALS AND METHODS}

We used the experimental protocol concerning the SMART kit PCR cDNA Synthesis (Clontech) on the candidate gene cDNA sequence.

We purified the polyA fraction and synthesized the first stalk of DNA with an oligodT.

A not specific amplification was performed and followed by a specific one with an oligo 956 forward ( $5^{\prime}$ 3') and then an oligo 957 reverse (5'-3').
The PCR products showed, in agarose gel, no band for the negative control and one band (400bp) for the specific PCR. This last was purified and sequenced on Illumina's GSII platform sequencing.

Experiences, in duplicate, were performed.

\section{RESULTS}

The results show that the revealed gene, after sequencing, postpones only little gene described in the previous paper (Swissprot database).

It is enough to decipher this gene to realize it: in here is the sequence, as following in the Fig. 1.

Now, we decipher the gene, with a higher probability by including the amino-acid sequence which is coded by DNA: here is this sequence (118 AA) in the Fig. 2 which is following.

\section{DISCUSSION}

By consulting Sander and Schneider (1991) review we think we can say that this gene is related to Immunoglobulins ( 2 typical cysteines of the domains of Ig appear in the sequence), without being able to assert, at the moment, if it is about a heavy or light chain of Immunoglobulins. A single indication (Delmotte et al., 2012) plead in the favor of a light chain.

Corresponding Author: Michel Leclerc, 556 Rue Isabelle Romée, Sandillon, France 
SMART_956.ab1:

tGACTGCTGCTATGCGTGGCAACATGGCGTCTCTATGGATGTTCTTCTTTGTCgTGG GGATAACTTTACAACGGAGTTTGGCGATTTACACGTTTCGCGAGCAACCGTCGGA CACTAGCGCGTTGCAGGGGAGCACAGTGGTGCTTCACTGCTCCGTTGAGCAGTAC ATAAACACCACGGCCATCGTTTGGTGGAGCCGTGACTCGGTCATCAGCCACAACA AAGACCTGAAACTGTCCAGTCTAAACACCGACCAGCTCCAAAGGTACTCGATTTC AGGCGACGCATCTCGGGGGGAATTCAACCTTAAAATAGTGAACTTTACCGNCACA GACGCCGCCAGTTACCGCTGTCAGATGTTTGCGA

Fig. 1. DNA Sequence of sea star IgKappa gene

\author{
MRGNMASLWM FFFVVGITLQ RSLAIYTFRE QPSDTSALQG STVVLHCSVE QYINTTAIVW \\ WSRDSVISHN KDLKLSSLNT DQLQRYSISG DASRGEFNLK IVNFTXTDAA SYRCQMFA
}

0209F30AE8E54D19 CRC64

Fig. 2. Sea star Igkappa gene including amino-acid sequence.(118 AA)

Otherwise «this sea star antibody» corresponds to HRP antigen, but could correspond as well to other antigens.

In fact, we don't know, at the moment, if the relation One antigen: One antibody is the rule in Invertebrates.

What we are sure, on the other hand: it is because there is well a gene (the sea star IgKappa), the function of which is the one of the defense of the sea star against the immunopathogenicity attacks: The HRP, in the present case. In terms of amino-acid, our gene could present 118 amino-acids as following (Fig. 2). We recall that the true IgKappa region V-IV S107B precursor gene found in metazoans and more particularly in mouse shows 129 amino-acids.

\section{CONCLUSION}

In conclusion, this antibody gene of sea star calls back the mouse IgKappa region V-IV S107B precursor gene but is different from it by the number of amino-acids (118 instead of 129 aa) and so by the DNA sequence sligthly different when compared to mouse precursor gene.

Insight into the molecular and genetic basis of nonself recognition by invertebrates. This will allow.

A much better comprehension of the evolution of MHC antigens and immunoglobulins through the animal world.

\section{REFERENCES}

Delmotte, F., C. Brillouet, M. Leclerc, G. Luquet and J.C. Kader, 2012. Purification of an antibody-like protein from the sea star Asterias rubens. Eur. J. Immunol., 16: 1325-1330. DOI: 10.1002/eji.1830161103

Leclerc, M. and C. Brillouet, 1981. Evidence of antibody-like substances secreted by axial organ cells of the starfish Asterias rubens. Immunol. Lett.. 2: 279-281. DOI: 10.1016/01652478(81)90020-1

Leclerc, M., 1973. Ultrastructural study of the reactions of Asterina Gilbbosa (echinoderm Ästende) at the axial body after injection of protein. Ann. Immunol., 124C: 363-374.

Leclerc, M., S. Dupont and B. Hernroth, 2011. Evidence of Kappa genes in the sea-star Asterias rubens (Echinoderma). Immunol. Lett., 138: 197-198. DOI: 10.1016/j.imlet.2011.01.016

Sander, C. and R. Schneider, 1991. Database of homology-derived protein structures and the structural meaning of sequence alignment. Proteins, 9: 56-68. DOI: 10.1002/prot.340090107 